\title{
RULED REAL HYPERSURFACES HAVING THE SAME SECTIONAL CURVATURE AS THAT OF AN AMBIENT NONFLAT COMPLEX SPACE FORM
}

\author{
Sadahiro Maeda, Hiromasa Tanabe and Young Ho Kim
}

\begin{abstract}
Ruled real hypersurfaces in a nonflat complex space form $\tilde{M}_{n}(c)(n \geqq 2)$ are obtained by having a one-codimensional foliation whose leaves are totally geodesic complex hypersurfaces of the ambient space. Motivated by a fact that the sectional curvature $K$ of every ruled real hypersurface $M$ in $\tilde{M}_{n}(c)(n \geqq 3)$ satisfies $|c / 4| \leqq$ $|K(X, Y)| \leqq|c|$ for an arbitrary pair of orthonormal vectors $X$ and $Y$ that are tangent to the leaf at each point $x$ of $M$, we study ruled real hypersurfaces having the sectional curvature $K$ with $|c / 4| \leqq|K| \leqq|c|$ in $\tilde{M}_{n}(c)$.
\end{abstract}

\section{Introduction}

We denote by $\tilde{M}_{n}(c)$ a complex $n$-dimensional complete and simply connected Kähler manifold of constant holomorphic sectional curvature $c(\neq 0)$ endowed with the standard Riemannian metric $\langle$,$\rangle and the Kähler structure$ $J$. This so-called an $n$-dimensional nonflat complex space form of constant holomorphic sectional curvature $c$ is holomorphically isometric to either an $n$-dimensional complex projective space $\mathbf{C} P^{n}(c)$ or an $n$-dimensional complex hyperbolic space $\mathbf{C H}^{n}(c)$ according as $c$ is positive or negative. We recall the fact that for $n \geqq 2$ the sectional curvatures $K$ of $\mathbf{C} P^{n}(c)$ and $\mathbf{C} H^{n}(c)$ satisfy $c / 4 \leqq K \leqq c$ and $c \leqq K \leqq c / 4$, respectively. Needless to say, the sectional curvatures $K$ of $\mathbf{C} P^{1}(c)$ and $\mathbf{C} H^{1}(c)$ satisfy $K=c$.

In this paper, among real hypersurfaces isometrically immersed into a nonflat complex space form $\tilde{M}_{n}(c)(n \geqq 2)$, we shall focus on ruled real hypersurfaces.

2010 Mathematics Subject Classification. Primary 53B25, Secondary 53C40.

Key words and phrases. ruled real hypersurfaces, sectional curvatures, nonflat complex space forms.

The first author is partially supported by Grant-in-Aid for Scientific Research (C) (No. 23540097), Japan Society for the Promotion of Sciences.

The third author is partially supported by Basic Science Research Program through the National Research Foundation of Korea (NRF) funded by the Ministry of Education, Science and Technology (2012R1A1A2042298).

Received February 24, 2015. 
In classical differential geometry, it is well-known that every ruled surface in 3-dimensional Euclidean space $\mathbf{R}^{3}$ has nonpositive Gaussian curvature. Hence we have an interest in the sectional curvature of every ruled real hypersurface $M$ in a nonflat complex space form $\tilde{M}_{n}(c)(n \geqq 2)$. Since every leaf of a onecodimensional foliation on $M$ is totally geodesic, in the case of $n \geqq 3$ we find easily the fact that the sectional curvature $K$ of every ruled real hypersurface $M$ in $\tilde{M}_{n}(c)$ satisfies $|c / 4| \leqq|K(X, Y)| \leqq|c|$ for an arbitrary pair of orthonormal vectors $X$ and $Y$ tangent to the leaf at each point $x$ of $M$. Motivated by this fact we study ruled real hypersurfaces $M$ having the sectional curvature $K$ with $|c / 4| \leqq|K| \leqq|c|$, that is, the sectional curvature $K$ satisfies either $c / 4 \leqq$ $K(X, Y) \leqq c(c>0)$ or $c \leqq K(X, Y) \leqq c / 4(c<0)$ for an arbitrary pair of orthonormal vectors $X$ and $Y$ that are not necessarily tangent to the leaf at each point $x$ of $M$.

Every ruled real hypersurface in $\tilde{M}_{n}(c)$ is constructed by attaching complex hyperplanes $\tilde{M}_{n-1}(c)$ on a smooth real curve $\gamma: I \rightarrow \tilde{M}_{n}(c)$ with its arclength $s$ defined on some open interval $I(\subset \mathbf{R})$ in such a way that the hyperplane $\tilde{M}_{n-1}(c)$ is orthogonal to the real plane spanned by $\{\dot{\gamma}(s), J \dot{\gamma}(s)\}$ at each point $\gamma(s)$. So, it is natural to investigate the relation between the properties of the curve $\gamma$ which generates a ruled real hypersurface $M$ and the value of the sectional curvature $K$ of $M$. In $\S 4$ we give our main results by using the notion of Frenet curves.

\section{Preliminaries}

Let $M^{2 n-1}$ be a real hypersurface of an $n(\geqq 2)$-dimensional nonflat complex space form $\tilde{M}_{n}(c)$ of constant holomorphic sectional curvature $c$ and $\mathscr{N}$ be a unit normal local vector field on $M$ in $\tilde{M}_{n}(c)$. Then the formulae of Gauss and Weingarten are given respectively by

$$
\tilde{\nabla}_{X} Y=\nabla_{X} Y+\langle A X, Y\rangle \mathscr{N}, \quad \tilde{\nabla}_{X} \mathscr{N}=-A X
$$

for vector fields $X$ and $Y$ tangent to $M$, where $\tilde{\nabla}$ and $\nabla$ denote the Riemannian connections of $\tilde{M}_{n}(c)$ and $M$, respectively, $\langle$,$\rangle denotes the Riemannian metric on$ $M$ induced from the standard metric on $\tilde{M}_{n}(c)$ and $A$ is the shape operator of $M$ in $\tilde{M}_{n}(c)$. The Kähler structure $J$ of $\tilde{M}_{n}(c)$ induces an almost contact metric structure $(\phi, \xi, \eta,\langle\rangle$,$) on the real hypersurface M$. That is, we define a tensor field $\phi$ of type $(1,1)$, a vector field $\xi$ and a 1 -form $\eta$ on $M$ by

$$
\xi=-J \mathscr{N}, \quad \eta(X)=\langle X, \xi\rangle=\langle J X, \mathscr{N}\rangle \quad \text { and } \quad \phi X=J X-\eta(X) \mathscr{N}
$$

for each tangent vector $X \in T M$. Then the structure satisfies

$$
\begin{gathered}
\phi^{2} X=-X+\eta(X) \xi, \quad\langle\phi X, \phi Y\rangle=\langle X, Y\rangle-\eta(X) \eta(Y), \\
\eta(\xi)=1, \quad \phi \xi=0 \quad \text { and } \quad \eta(\phi X)=0
\end{gathered}
$$


for all vectors $X, Y \in T M$. We call the vector field $\xi$ the characteristic vector field on $M$. It follows from the equalities (2.1) and $\tilde{\nabla} J=0$ that

$$
\left(\nabla_{X} \phi\right) Y=\eta(Y) A X-\langle A X, Y\rangle \xi
$$

and

$$
\nabla_{X} \xi=\phi A X .
$$

Denoting the curvature tensor of $M$ by $R$, we have the equation of Gauss given by

$$
\begin{aligned}
& \langle R(X, Y) Z, W\rangle \\
& =(c / 4)\{\langle Y, Z\rangle\langle X, W\rangle-\langle X, Z\rangle\langle Y, W\rangle \\
& +\langle\phi Y, Z\rangle\langle\phi X, W\rangle-\langle\phi X, Z\rangle\langle\phi Y, W\rangle \\
& \quad-2\langle\phi X, Y\rangle\langle\phi Z, W\rangle\} \\
& +\langle A Y, Z\rangle\langle A X, W\rangle-\langle A X, Z\rangle\langle A Y, W\rangle
\end{aligned}
$$

for vector fields $X, Y, Z, W$ on $M$.

For a real hypersurface $M$ in $\tilde{M}_{n}(c)(n \geqq 2)$, the condition that $\eta=0$ defines a $(2 n-2)$-dimensional subbundle $T^{0} M$ of the tangent bundle $T M$ called the holomorphic distribution: $T^{0} M=\bigcup_{x \in M} T_{x}^{0} M$, where $T_{x}^{0} M=\left\{X \in T_{x} M\right.$ $\eta(X)=\langle X, \xi\rangle=0\}$. A real hypersurface $M$ in $\tilde{M}_{n}(c)$ is said to be ruled if the holomorphic distribution $T^{0} M$ is integrable and each of its leaves (i.e., maximal integral manifolds) is a totally geodesic complex hypersurface $\tilde{M}_{n-1}(c)$ of $\tilde{M}_{n}(c)$. The construction of ruled real hypersurfaces in Introduction means that in general a ruled real hypersurface has singularities. So we must omit such points.

We define two functions $\mu, v: M \rightarrow \mathbf{R}$ by $\mu=\langle A \xi, \xi\rangle$ and $v=\|A \xi-\mu \xi\|$. These functions $\mu$ and $v$ are important quantities which measure how far the characteristic vector field $\xi$ is from being a principal curvature vector. A characterization of ruled real hypersurfaces in terms of the functions $\mu, v$ and the shape operator $A$ is given as follows.

Proposition 1 ([8]). Let $M$ be a real hypersurface in a nonflat complex space form $\tilde{M}_{n}(c)(n \geqq 2)$. Then the following three conditions are mutually equivalent.

(1) $M$ is a ruled real hypersurface.

(2) The shape operator $A$ of $M$ satisfies $\langle A X, Y\rangle=0$ for any tangent vectors $X, Y \in T_{x} M$ orthogonal to $\xi_{x}$ at each point $x \in M$.

(3) The set $M_{1}=\{x \in M \mid v(x) \neq 0\}$ is an open dense subset of $M$ and there exists a unit vector field $U$ on $M_{1}$ such that it is orthogonal to $\xi$ and satisfies that

$$
A \xi=\mu \xi+v U, \quad A U=v \xi \quad \text { and } \quad A X=0
$$

for an arbitrary tangent vector $X$ orthogonal to both $\xi$ and $U$. 
In this paper, considering a ruled real hypersurface $M$, we treat $M$ as a differentiable manifold such that $M=M_{1} \cup\{x \in M \mid v(x)=0\}$.

\section{Sectional curvatures of ruled real hypersurfaces}

Let $K$ be the sectional curvature of a ruled real hypersurface $M$ in a nonflat complex space form $\tilde{M}_{n}(c)(n \geqq 2)$. That is, $K$ is defined by $K(X, Y)=$ $\langle R(X, Y) Y, X\rangle$ for orthonormal tangent vectors $X, Y$ on $M$ with the curvature tensor $R$ of $M$.

First of all we give the following inequalities for the sectional curvature $K$ of a ruled real hypersurface $M(c f$. [9]).

Lemma 1. Let $M$ be a ruled real hypersurface in $\tilde{M}_{n}(c)(n \geqq 2)$. Then the sectional curvature $K$ of $M$ satisfies the following:

(1) If $c>0$, we have $(c / 4)-v(x)^{2} \leqq K \leqq c$ at every point $x \in M$;

(2) If $c<0$, we have $(c / 4)-v(x)^{\frac{2}{2}} \leqq K \leqq c / 4$ at the point $x \in M$ with $v(x)^{2} \geqq 3|c| / 4$ and $c \leqq K \leqq c / 4$ at the point $x \in M$ with $v(x)^{2}<3|c| / 4$. These estimates are sharp in the sense that at each point of $M$ we can take a pair of orthonormal tangent vectors $X$ and $Y$ satisfying $K(X, Y)=k$ for given $k$ with $K_{\min } \leqq k \leqq K_{\max }$

Proof. By the equation of Gauss (2.6), the sectional curvature $K$ is given as

$$
K(X, Y)=(c / 4)\left\{1+3\langle\phi X, Y\rangle^{2}\right\}+\langle A X, X\rangle\langle A Y, Y\rangle-\langle A X, Y\rangle^{2}
$$

for orthonormal tangent vectors $X, Y$ on $M$. At a point $x$ of $M$, we take an arbitrary 2-dimensional vector subspace $P$ of the tangent space $T_{x} M$. Then we can choose an orthonormal pair of vectors $X, Y \in T_{x}^{0} M$ and a real number $t$ such that vectors $\cos t \cdot \xi_{x}+\sin t \cdot X$ and $Y$ form the orthonormal basis of the subspace $P$.

First, we examine the case where $v(x) \neq 0$. By using (2.3) and Proposition $1(2)$, (3), we find the sectional curvature $K$ of the plane section $P$ can be written as

$$
\begin{aligned}
& K\left(\cos t \cdot \xi_{x}+\sin t \cdot X, Y\right) \\
& \quad=(c / 4)\left\{1+3 \sin ^{2} t\langle\phi X, Y\rangle^{2}\right\}-v(x)^{2} \cos ^{2} t\left\langle Y, U_{x}\right\rangle^{2} .
\end{aligned}
$$

If $c>0$, we can see that the expression of (3.2) takes its maximum value $c$ at $t=\pi / 2$ and $X=\phi Y$ (that is, for example, in the case that a plane section $P$ is a $\phi$-section spanned by $U_{x}$ and $\phi U_{x}$ ). Also, it takes the minimum value $(c / 4)-v(x)^{2}$ at $t=0, Y=U_{x}$ (that is, in the case that the plane $P$ is spanned by $U_{x}$ and $\left.\xi_{x}\right)$. If $c<0$, it follows from (3.2) that

$$
\begin{aligned}
K\left(\cos t \cdot \xi_{x}+\sin t \cdot X, Y\right) & \geqq(c / 4)\left(1+3 \sin ^{2} t\right)-v(x)^{2} \cos ^{2} t \\
& =(c / 4)-v(x)^{2}+\left((3 c / 4)+v(x)^{2}\right) \sin ^{2} t
\end{aligned}
$$


Hence, if $(3 c / 4)+v(x)^{2} \geqq 0$, the sectional curvature $K$ takes its minimum value $(c / 4)-v(x)^{2}$ at $t=0, Y=U_{x}, X=\phi U_{x}$. If $(3 c / 4)+v(x)^{2}<0$, it takes the minimum value $(c / 4)-v(x)^{2}+\left\{(3 c / 4)+v(x)^{2}\right\}=c$ at $t=\pi / 2, \quad Y=U_{x}, X=$ $\phi U_{x}$. Obviously, we find that the maximum value of $K$ is $c / 4$ from (3.2).

Next we consider the case where $v(x)=0$. Since $A \xi_{x}=\mu(x) \xi_{x}$ by the definition of $v$, we see from Proposition 1(2)

$$
K\left(\cos t \cdot \xi_{x}+\sin t \cdot X, Y\right)=(c / 4)\left\{1+3 \sin ^{2} t\langle\phi X, Y\rangle^{2}\right\} .
$$

This implies our inequalities are valid at the point $x$ with $v(x)=0$. Thus we have the assertion (2). The last assertion of the lemma is clear from the above argument.

\section{Ruled real hypersurfaces associated with Frenet curves}

Take an arbitrary regular real curve $\gamma: I \rightarrow \tilde{M}_{n}(c)$ with its arclength $s$ defined on some open interval $I(\subset \mathbf{R})$. Then we consider a ruled real hypersurface $M=\bigcup_{s \in I} \tilde{M}_{n-1}^{(s)}(c)$ associated with the curve $\gamma$ with arclength $s$, where $\tilde{M}_{n-1}^{(s)}(c)$ is a complex hyperplane through the point $\gamma(s)$ (for details, see Introduction). Needless to say that the curve $\gamma$ is an integral curve of the characteristic vector field $\xi$ of $M$.

In [3], Lohnherr-Reckziegel studied ruled real hypersurfaces by parameterizing them by maps of the form $f: I \times \tilde{M}_{n-1}(c) \rightarrow \tilde{M}_{n}(c)$ and show properties on these maps. The following lemma is due to them.

Lemma 2 ([3]). For every $s \in I$ the function $v$ satisfies the following:

(1) When $c>0$, on each leaf $\tilde{M}_{n-1}^{(s)}(c)$ we have $0 \leqq v<\infty$;

(2) When $c<0$, on each leaf $\tilde{M}_{n-1}^{(s)}(c)$ we have either $0 \leqq v<\sqrt{|c|} / 2$, $v \equiv \sqrt{|c|} / 2$ or $\sqrt{|c|} / 2<v<\infty$.

Here we recall the definition of Frenet curves in a Riemannian manifold $\tilde{M}$. A smooth curve $\gamma=\gamma(s)$ parametrized by its arclength $s$ is called a Frenet curve of proper order $d$ if there exist a field of orthonormal frames $\left\{V_{1}=\dot{\gamma}, V_{2}, \ldots, V_{d}\right\}$ along $\gamma$ and positive smooth functions $\kappa_{1}(s), \ldots, \kappa_{d-1}(s)$ satisfying the following system of ordinary differential equations

$$
\tilde{\nabla}_{\dot{\gamma}} V_{j}(s)=-\kappa_{j-1}(s) V_{j-1}(s)+\kappa_{j}(s) V_{j+1}(s), \quad j=1, \ldots, d,
$$

where $V_{0} \equiv V_{d+1} \equiv 0$ and $\tilde{\nabla}_{\dot{\gamma}}$ denotes the covariant differentiation along $\gamma$ with respect to the Riemannian connection $\tilde{\nabla}$ of $\tilde{M}$. The functions $\kappa_{j}(s)(j=1, \ldots$, $d-1)$ and a field of orthonormal frames $\left\{V_{1}, \ldots, V_{d}\right\}$ are called the curvatures and the Frenet frame of $\gamma$, respectively. We call a curve a helix when all of its curvatures are constant functions. A curve $\gamma$ is called a helix of order $d$ if it is a helix of proper order $r(\leqq d)$. For a helix of order $d$, which is of proper order $r(\leqq d)$, we use the convention in (4.1) that $\kappa_{j}=0(r \leqq j \leqq d-1)$ and $V_{j}=0$ $(r+1 \leqq j \leqq d)$. A helix of order 1 is nothing but a geodesic. A helix of order 
2, that is a curve which satisfies $\tilde{\nabla}_{\dot{\gamma}} \dot{\gamma}(s)=k V_{2}(s), \tilde{\nabla}_{\dot{\gamma}} V_{2}(s)=-k \dot{\gamma}(s)$, is called a circle of curvature $k(\geqq 0)$.

For a Frenet curve $\gamma$ of proper order $d$ in a nonflat complex space form $\tilde{M}_{n}(c)$, we define its complex torsions by $\tau_{i j}(s)=\left\langle V_{i}(s), J V_{j}(s)\right\rangle(1 \leqq i<j \leqq d)$. In the study of Frenet curves in $\tilde{M}_{n}(c)$ their complex torsions play an important role (see [6]). A circle of positive curvature in $\tilde{M}_{n}(c)$ is said to be totally real if its complex torsion $\tau_{12}$ vanishes. A totally real circle lies on a totally real totally geodesic surface $M^{2}(c)\left(=\mathbf{R} P^{2}(c / 4)\right.$ or $\left.\mathbf{R} H^{2}(c / 4)\right)$.

Now, we consider a ruled real hypersurface associated with a Frenet curve $\gamma$ and investigate the value of the function $v$ along $\gamma$.

LEMma 3. Let $\gamma=\gamma(s)$ be a Frenet curve of proper order $d$ with curvatures $\kappa_{j}(s)(1 \leqq j \leqq d-1)$ and complex torsions $\tau_{i j}(s)(1 \leqq i<j \leqq d)$ in a nonflat complex space form $\tilde{M}_{n}(c), n \geqq 2$ and $M$ be a ruled real hypersurface associated with the curve $\gamma$. Then the functions $\mu=\langle A \xi, \xi\rangle$ and $v=\|A \xi-\mu \xi\|$ satisfy the following.

$$
\begin{gathered}
\mu(\gamma(s))=-\kappa_{1}(s) \tau_{12}(s), \\
v(\gamma(s))^{2}=\kappa_{1}(s)^{2}\left(1-\tau_{12}(s)^{2}\right) .
\end{gathered}
$$

Proof. As $\dot{\gamma}(s)=\xi_{\gamma(s)}$, we see from (2.1) and (2.5) that

$$
\begin{aligned}
\tilde{\nabla}_{\dot{\gamma}} \dot{\gamma}(s) & =\nabla_{\dot{\gamma}} \dot{\gamma}(s)+\langle A \dot{\gamma}(s), \dot{\gamma}(s)\rangle \mathscr{N}_{\gamma(s)} \\
& =\phi A \xi_{\gamma(s)}+\mu(\gamma(s)) \mathscr{N}_{\gamma(s)} .
\end{aligned}
$$

We consider firstly the case in which $v(\gamma(s)) \neq 0$. By use of (2.3) and (2.7) we have

$$
\begin{aligned}
\tilde{\nabla}_{\dot{\gamma}} \dot{\gamma}(s) & =\phi\left\{\mu(\gamma(s)) \xi_{\gamma(s)}+v(\gamma(s)) U_{\gamma(s)}\right\}+\mu(\gamma(s)) \mathscr{N}_{\gamma(s)} \\
& =v(\gamma(s)) \phi U_{\gamma(s)}+\mu(\gamma(s)) \mathscr{N}_{\gamma(s)},
\end{aligned}
$$

so that the first curvature $\kappa_{1}(s)$ of $\gamma$, considered as a curve in $\tilde{M}_{n}(c)$, satisfies

$$
\kappa_{1}(s)^{2}=v(\gamma(s))^{2}+\mu(\gamma(s))^{2}(>0)
$$

and

$$
V_{2}(s)=\left(1 / \kappa_{1}(s)\right)\left\{v(\gamma(s)) \phi U_{\gamma(s)}+\mu(\gamma(s)) \mathscr{N}_{\gamma(s)}\right\}
$$

This gives

$$
\begin{aligned}
\tau_{12}(s) & =\left\langle\dot{\gamma}(s), J V_{2}(s)\right\rangle \\
& =\left(1 / \kappa_{1}(s)\right)\left\langle\xi_{\gamma(s)}, J\left\{v(\gamma(s)) \phi U_{\gamma(s)}+\mu(\gamma(s)) \mathscr{N}_{\gamma(s)}\right\}\right\rangle \\
& =-\left(1 / \kappa_{1}(s)\right)\left\langle\mathscr{N}_{\gamma}(s), v(\gamma(s)) \phi U_{\gamma(s)}+\mu(\gamma(s)) \mathscr{N}_{\gamma(s)}\right\rangle \\
& =-\mu(\gamma(s)) / \kappa_{1}(s) .
\end{aligned}
$$

Thus we obtain (4.2), which, together with (4.5), implies (4.3). 
Next, let us consider the case in which there exists some $s_{0} \in I$ such that $v\left(\gamma\left(s_{0}\right)\right)=0$. Then we have $A \xi_{\gamma\left(s_{0}\right)}=\mu\left(\gamma\left(s_{0}\right)\right) \xi_{\gamma\left(s_{0}\right)}$ and hence equation (4.4) becomes

$$
\left(\tilde{\nabla}_{\dot{\gamma}} \dot{\gamma}\right)\left(s_{0}\right)=\mu\left(\gamma\left(s_{0}\right)\right) \mathscr{N}_{\gamma\left(s_{0}\right)} .
$$

If $\mu\left(\gamma\left(s_{0}\right)\right)>0$, we find $\kappa_{1}\left(s_{0}\right)=\mu\left(\gamma\left(s_{0}\right)\right), V_{2}\left(s_{0}\right)=\mathscr{N}_{\gamma\left(s_{0}\right)}$ and therefore

$$
\tau_{12}\left(s_{0}\right)=\left\langle\dot{\gamma}\left(s_{0}\right), J V_{2}\left(s_{0}\right)\right\rangle=\left\langle\xi_{\gamma\left(s_{0}\right)}, J \mathscr{N}_{\gamma\left(s_{0}\right)}\right\rangle=-1 .
$$

Relations (4.2) and (4.3) follow from this. If $\mu\left(\gamma\left(s_{0}\right)\right)=0$, equation (4.6) shows $\kappa_{1}\left(s_{0}\right)=0$, so relations (4.2) and (4.3) hold. If $\mu\left(\gamma\left(s_{0}\right)\right)<0$, from (4.6) we have $\kappa_{1}\left(s_{0}\right)=-\mu\left(\gamma\left(s_{0}\right)\right), V_{2}\left(s_{0}\right)=-\mathscr{N}_{\gamma\left(s_{0}\right)}$. Then, just as above we get the conclusion.

As a direct consequence of Lemmas 1,2 and 3, we have the following.

THEOREM 1. Every ruled real hypersurface $M$ of a complex projective space $\mathbf{C} P^{n}(c)(n \geqq 2)$ has the sectional curvature $K$ with $-\infty<K \leqq c$, so that there does not exist a ruled real hypersurface having the sectional curvature $K$ with $c / 4 \leqq K \leqq c$ in this ambient space.

Theorem 2. Let $\gamma: I \rightarrow \mathbf{C} H^{n}(c)(n \geqq 2)$ be a Frenet curve of proper order $d$ defined on an open interval $I(\subset \mathbf{R})$ in a complex hyperbolic space $\mathbf{C} H^{n}(c)$ and $M$ be a ruled real hypersurface associated with the curve $\gamma$ in $\mathbf{C H}(c)$. Then $M$ has the sectional curvature $K$ with $c \leqq K \leqq c / 4$ if and only if the first curvature $\kappa_{1}$ and a complex torsion $\tau_{12}$ of the Frenet curve $\gamma$ satisfy $\kappa_{1}(s)^{2}\left(1-\tau_{12}(s)^{2}\right) \leqq|c| / 4$ for any $s \in I$.

Remark 1. As a matter of course, the curve $\gamma$ which generates a ruled real hypersurface $M$ is not unique. At any point $p$ of $M$ there exists the unique curve $\gamma_{p}$ through $p$, which generates $M$. It follows from Lemma 2(2) and (4.3) that in Theorem 2 the inequality $\kappa_{1}(s)^{2}\left(1-\tau_{12}(s)^{2}\right) \leqq|c| / 4$ holds on every $\gamma$ generating $M$.

\section{Examples}

Example 1. In [2], Adachi, Bao and the first author studied congruency of minimal ruled real hypersurfaces in a nonflat complex space form $\tilde{M}_{n}(c), n \geqq 2$ with respect to the action of its isometry group $\mathrm{I}\left(\tilde{M}_{n}(c)\right)$. They showed that those in a complex projective space $\mathbf{C} P^{n}(c)(n \geqq 2)$ are congruent to each other and that those in a complex hyperbolic space $\mathbf{C} H^{n}(c)(n \geqq 2)$ are classified into three classes.

Here we consider minimal ruled real hypersurface $M$ in a complex hyperbolic space. It is known that every minimal ruled real hypersurface $M$ is associated with a totally real circle $\gamma$ and vice versa $([3,4])$. Moreover, on the 
minimal ruled real hypersurface $M$ the function $\mu$ vanishes identically, the function $v$ is constant along each integral curve of its characteristic vector field $\xi$ and we have $\tilde{\nabla}_{\dot{\gamma}} \dot{\gamma}(s)=v(\gamma(s)) \phi U_{\gamma(s)}, \tilde{\nabla}_{\dot{\gamma}}(\phi U)_{\gamma(s)}=-v(\gamma(s)) \dot{\gamma}(s)$ with $\dot{\gamma}(s)=\xi_{\gamma(s)}$. These equations mean that a totally real circle $\gamma$ generating a minimal ruled real hypersurface $M$ has just one curvature $k=v(\gamma(s))$ along the curve $\gamma$. On the other hand, totally real circles in $\mathbf{C H}(c)$ are classified into three kind of classes according to their curvatures ([1]). If the curvature $k$ of a totally real circle $\gamma$ is greater than $\sqrt{|c|} / 2, \gamma$ is closed and bounded. If $k=\sqrt{|c|} / 2$, it is horocyclic, so that it is unbounded and has a single limit point at infinity $\gamma(\infty)=\gamma(-\infty)$ in the ideal boundary of $\mathbf{C} H^{n}(c)$. If $0 \leqq k<\sqrt{|c|} / 2$, it is unbounded and has two distinct limit points at infinity $\gamma(\infty), \gamma(-\infty)$ in the ideal boundary. Corresponding to this classification of totally real circles, we call ruled real hypersurfaces $M$ associated with $\gamma$ of elliptic type, parabolic type and axial type according as the curvature of $\gamma$ satisfies $k>\sqrt{|c|} / 2, k=\sqrt{|c|} / 2$ and $0 \leqq k<$ $\sqrt{|c|} / 2$ ([2]). Every minimal ruled real hypersurface is locally congruent to one of minimal ruled real hypersurfaces of axial type, parabolic type and elliptic type. Minimal ruled real hypersurfaces of parabolic type and axial type are complete, but minimal ruled real hypersurfaces of elliptic type are not complete. Furthermore, the minimal ruled real hypersurface of parabolic type is homogeneous, i.e. this minimal ruled real hypersurface is an orbit of some subgroup of the full isometry group of the ambient space $\mathbf{C} H^{n}(c)$. Note that the isometry group of the minimal ruled real hypersurface of parabolic type is the direct product of $\mathrm{I}\left(\mathrm{CH}^{n-1}(c)\right)$ and a one-parameter subgroup whose orbit is a horocycle on a totally geodesic $\mathbf{R} H^{2}(c / 4)$, where $\mathrm{I}\left(\mathbf{C} H^{n-1}(c)\right)$ is the isometry group of a totally geodesic $\mathbf{C} H^{n-1}(c)$ (for details, see $[2,5]$ ).

Returning to our main topics, since the complex torsion $\tau_{12}$ of a totally real circle $\gamma$ vanishes, by Theorem 2 we can see that minimal ruled real hypersurfaces of types axial and parabolic in $\mathbf{C H}^{n}(c)$ have the sectional curvature $K$ with $c \leqq K \leqq c / 4$. In particular, the minimal ruled real hypersurface associated with geodesics on a totally geodesic $\mathbf{R} H^{2}(c / 4)$ has the sectional curvature $K$ with $c \leqq K \leqq c / 4$.

Remark 2. For the minimal ruled real hypersurface of elliptic type we have $-\infty<K \leqq c / 4$.

Example 2. Take an arbitrary geodesic $\gamma$ in $\mathbf{C} H^{n}(c)(n \geqq 2)$ and consider the ruled real hypersurface $M$ associated with $\gamma$. Then we find $\kappa_{1} \equiv 0$, so that $M$ has the sectional curvature $K$ with $c \leqq K \leqq c / 4$ by Theorem 2 . However this ruled real hypersurface $M$ can be considered the minimal ruled real hypersurface of axial type. In fact, since $\mathbf{C H}^{n}(c)$ is a Riemannian symmetric space of rank one, our geodesic $\gamma$ is congruent to a geodesic on $\mathbf{R} H^{2}(c / 4)$.

Example 3. Let $M$ be a ruled real hypersurface in $\mathrm{CH}^{n}(c)(n \geqq 2)$ associated with a curve $\gamma=\gamma(s)$ lying on a totally geodesic complex line $\mathbf{C} H^{1}(c)$. Then the curve $\gamma$ satisfies $\tilde{\nabla}_{\dot{\gamma}} \dot{\gamma}(s)=\kappa(s) J \dot{\gamma}(s)$ for each $s \in I$, where 
$\kappa=\kappa(s)(\geqq 0)$ is a smooth function along $\gamma$. Such a curve is called Kähler Frenet curve ([7]). Needless to say, every geodesic is a Kähler Frenet curve in a trivial sense. We can find $\left|\tau_{12}\right|=1$ on the curve $\gamma$ and hence by Theorem $2 M$ has the sectional curvature $K$ with $c \leqq K \leqq c / 4$. We here remark that the characteristic vector $\xi$ is principal along the above curve $\gamma$. But, in general the vector $\xi$ is not principal along other curves generating $M$, that is, $\tau_{12} \neq \pm 1$ on these curves. (In fact, if $\tau_{12}= \pm 1$ on every curve generating $M$, then we see easily our ruled real hypersurface $M$ is a Hopf hypersurface, that is, the characteristic vector $\xi$ on $M$ is principal at its each point. This is a contradiction.) However the inequality in Theorem 2 must hold on every curve generating $M$.

\title{
REFERENCES
}

[1] T. Adachi and S. Maeda, Global behaviours of circles in a complex hyperbolic space, Tsukuba J. Math. 21 (1997), 29-42.

[2] T. Adachi, T. BaO And S. Maeda, Congruence classes of minimal ruled real hypersurfaces in a nonflat complex space form, Hokkaido Math. J. 43 (2014), 1-14.

[ 3 ] M. LOHNHERR AND H. ReCKziegel, On ruled real hypersurfaces in complex space forms, Geom. Dedicata 79 (1999), 267-286.

[ 4 ] S. MAEDA AND T. ADACHI, Integral curves of characteristic vector fields of real hypersurfaces in nonflat complex space forms, Geom. Dedicata 123 (2006), 65-72.

[5] S. Maeda, T. Adachi AND Y. H. Kim, A characterization of the homogeneous minimal ruled real hypersurface in a complex hyperbolic space, J. Math. Soc. Japan 61 (2009), 315-325.

[6] S. Maeda AND Y. Ohnita, Helical geodesic immersions into complex space forms, Geom. Dedicata 30 (1989), 93-114.

[ 7 ] S. Maeda AND H. Tanabe, Totally geodesic immersion of Kähler manifolds and Kähler Frenet curves, Math. Z. 252 (2006), 787-795.

[8] R. Niebergall and P. J. Ryan, Real hypersurfaces in complex space forms, Tight and taut submanifolds (T. E. Cecil and S. S. Chern, eds.), Cambridge Univ. Press, 1998, 233-305.

[9] H. TANABe AND S. MaEda, Sectional curvatures of ruled real hypersurfaces in a nonflat complex space form, Proceedings of the workshop on Differential Geometry of Submanifolds and its related topics, Saga, August 4-6, 2012, World Scientific, 2013, 113-118.

\author{
Sadahiro Maeda \\ Department of Mathematics \\ SAGA UNIVERSITy \\ 1 HonZyo, SAGA, 840-8502 \\ JAPAN \\ E-mail: smaeda@ms.saga-u.ac.jp \\ Hiromasa Tanabe \\ Department of Science \\ National Institute of TeChNology \\ Matsue College \\ Matsue, Shimane 690-8518 \\ JAPAN \\ E-mail: h-tanabe@matsue-ct.jp
}


Young Ho Kim

DePARTMENT OF MATHEMATICS

KYUNGPOOK NATIONAL UNIVERSITY

TAEGU 702-701

KorEA

E-mail: yhkim@knu.ac.kr 